\title{
SIVOR: A TESTBED FOR THE EVALUATION OF ON-OFF VALVES ON AN F16 AIRPLANE - VIABILITY ANALYSIS
}

\author{
Guilherme Sartori Natal \\ Instituto Tecnológico de \\ Aeronáutica \\ São José dos Campos, SP, Brazil
}

\author{
Emilia Villani \\ Instituto Tecnológico de \\ Aeronáutica \\ São José dos Campos, SP, Brazil
}

\author{
Petter Krus \\ Linköping University - \\ $\mathrm{LiU}$ \\ Linköping, Sweden
}

\begin{abstract}
In this paper, the viability for the utilization of the SIVOR (Simulador de Voo Robótico - Robotic Flight Simulator) as a testbed for the evaluation of the dynamics changes caused by the replacement of conventional servovalves by on-off valves on an F16 airplane will be analyzed. The current version of the SIVOR consists of an industrial robotic arm that carries a cabin which contains a seat, a curved 55-inches TV, a joystick, a throttle stick, pedals and a black cover to avoid external interferences from the peripheral vision, which can break the immersion of the simulation. The replacement of conventional servovalves by on-off valves has been considered in order to increase the energy efficiency of the airplane hydraulic actuators. However, on-off valves might cause undesirable effects on the flight dynamics. Therefore, this replacement must be adequately evaluated through simulations before being tested on an actual airplane. In order to conclude if the SIVOR is a viable solution for this analysis or not, the necessary workspace for the simulation to be representative will be defined. This study will consist of a comparison between the linear accelerations and angular velocities sensed by a pilot inside the airplane and inside the SIVOR cabin, which are estimated through vestibular system models. The SIVOR will be considered viable if it is able to provide a workspace that generates a small enough motion sensation error between the F16 airplane and the simulator cabin.
\end{abstract}

Keywords: Robotics, Aircraft actuation systems, Efficiency, On/off valves, Hydraulic systems.

\section{INTRODUCTION}

The replacement of conventional servovalves by on-off valves has been considered in several applications. For instance, the actuators of airplane control surfaces. The main reason is the lack of energetic efficiency of the servovalves [1]. Over the past few years, several studies have investigated increasing the efficiency of hydraulic systems. One approach with particular potential is the use of digital hydraulics. Although many ideas regarding digital hydraulics were presented decades ago, such research has only achieved significant development in recent years [2]. The main advantages of digital hydraulics include not only higher efficiency, but also redundancy, robustness, among others [3]-[6].

However, the effects that such replacement would have on the controllability of an airplane have not yet been investigated. In order to maximize the safety of such investigation, the analysis of the viability for the utilization of the SIVOR platform [7] is proposed in this study.

The present work is organized as follows. A description of the SIVOR platform is presented in the following section. An overview of the algorithm that converts the dynamics of the F16 airplane to the dynamics of the robot will be detailed in the "Washout Filter" section. The vestibular models used to estimate the pilot's sensations in the airplane and in the SIVOR platform will be introduced in the "Vestibular Models" section. The comparison between the pilot's sensations in the airplane and in the SIVOR platform for both configurations of the F16 airplane (with servovalves and on-off valves) will be made in the "Results" section. Finally, the conclusions will be presented in the "Conclusions" section.

\section{THE SIVOR PLATFORM}

The current and the future versions of the SIVOR prototype are illustrated in Figure 1. The current version is composed of an industrial 6-DOF anthropomorphic robot with 50okg payload capacity and a single seat attached to it as its end-effector. Onboard, the pilot has the essential aircraft commands, such as sidestick, pedals, power lever, flaps selector, among others. The visual system consists of a single monitor. A blackout cover reduces external disturbances. The future version will consist of an industrial 6-DOF anthropomorphic robot with 100okg payload capacity mounted over a 10m long linear axis (making it a 7-DOF robotic platform), a cabin with actual airplane-like dimensions and inceptors, as well as a screen that covers $180^{\circ}$ horizontal and $40^{\circ}$ vertical field of view of the pilots. 


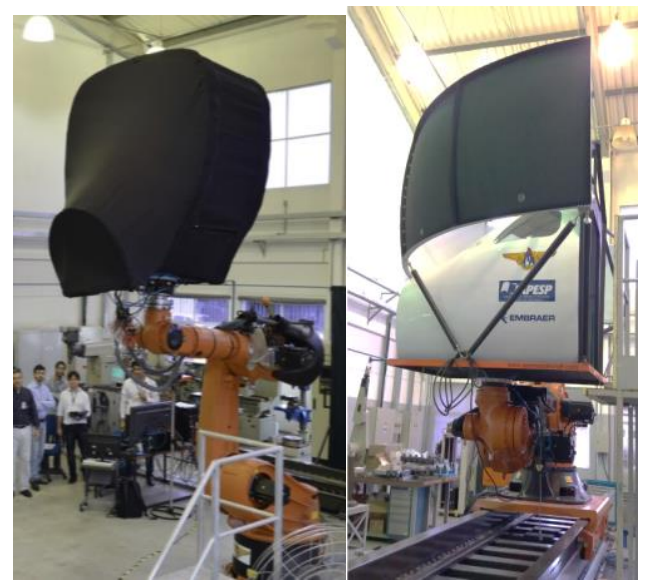

Figure 2 - Current version of the SIVOR platform (left), future version (right)

\section{WASHOUT FILTER}

The washout filter [8] is the algorithm that transforms the airplane dynamics into the robot dynamics, such that the pilot has similar motion sensations, in the simulator, when compared to those he/she has on the real airplane. It is organized in three channels that generate the desired Cartesian trajectory that the robot must track, as depicted in Figure 2.

The translational channel defines the translational movements of the end-effector. It receives as input the linear accelerations of the aircraft, measured at the estimated position of the pilot's head using a coordinate system attached to the aircraft CG, passes the high frequency components of its input signals and integrates them in order to estimate the robot position.

The tilt coordination channel passes the low frequency components of the linear accelerations and converts them to angular positions. The concept behind this channel is to use the projection of the gravity acceleration (G) to emulate a sustained linear acceleration.

Finally, the rotational channel processes the cockpit angular velocities. It converts the airplane angular velocities to end-effector angular velocities by passing the high frequency components of the airplane angular velocities and integrating them in order to obtain angular positions. The final desired orientation of the end-effector is obtained by the addition of these angular positions to the ones obtained through the tilt coordination channel.

\section{VESTIBULAR MODELS}

In an attempt to quantify and compare pilots' motion perceptions in the airplane and in the SIVOR platform, the vestibular models used in the present work are given as follows [9]:

$$
H_{\text {OTO }}=\frac{0.4(13.2 s+1)}{(5.33 s+1)(0.66 s+1)}
$$

for the otolith, which senses linear forces, and

$$
H_{S S C}=\frac{456 s^{2}}{(5.7 s+1)(80 s+1)}
$$

for the semicircular canal, which senses angular velocities [10]. The inputs of these transfer functions will be the linear accelerations and angular velocities of the airplane, respectively, as well as the equivalent outputs of the washout filter (SIVOR platform desired trajectories), for comparison purposes. The output of the vestibular models to the airplane dynamics and to the SIVOR platform dynamics will be compared in order to define if the SIVOR platform is expected to provide a good enough motion realism to the pilots or not. 


\section{RESULTS}

In the present work, the behavior of the F16 airplane to altitude commands was evaluated for both F16 hydraulics system configurations (using servovalves and on-off valves). The performed flights with the servovalves and the on-off valves are displayed in Figure 3.

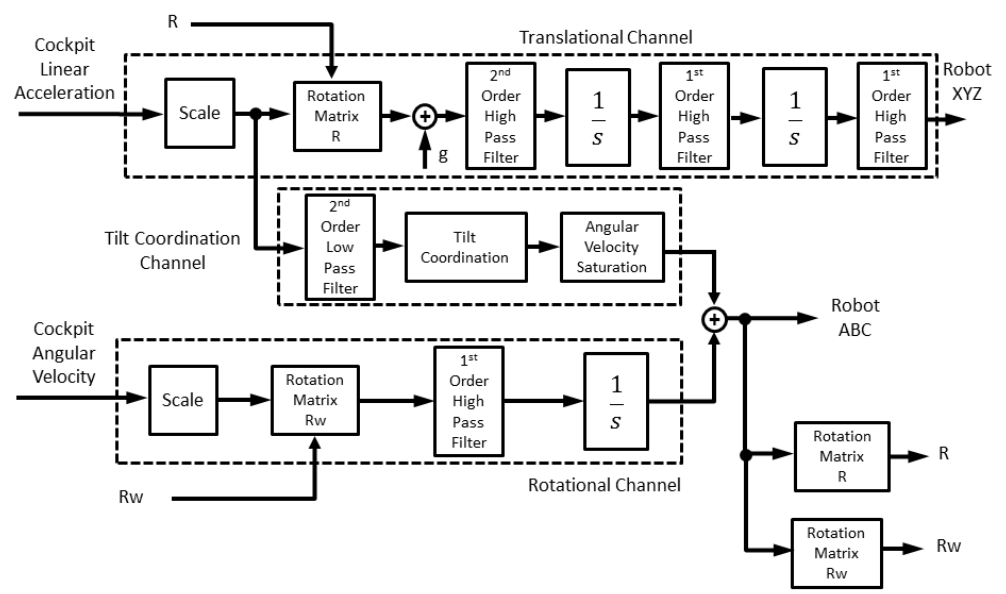

Figure 2 - Block diagram of the classical washout filter

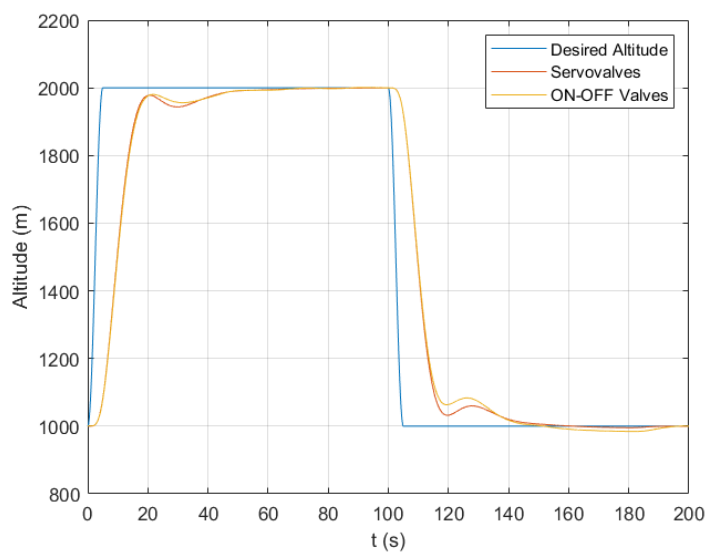

Figure 3 - F16 performed flight with servovalves and on-off valves

The analyzed washout outputs (which become the SIVOR inputs) were the translations in the $\mathrm{Z}$ axis (vertical) and the rotations around the $\mathrm{B}$ axis (equivalent to the pitch in the airplane). The $\mathrm{Z}$ axis' translation range goes from $1.5 \mathrm{~m}$ to $1.5 \mathrm{~m}$ and the $\mathrm{B}$ axis' rotation range goes from $-40^{\circ}$ to $40^{\circ}$. A comparison between the outputs of the vestibular system models to the same flight performed in the airplane and in the SIVOR platform, as well as the illustration of the SIVOR platform workspace usage in these flights are provided in Figures 4 (servovalves setup) and 5 (on-off valves setup).

It is possible to notice that in both cases the SIVOR platform can generate motion sensations that are similar to those the pilot would have in the real airplane, without reaching the limits of the robot's workspace. It is also possible to notice that the pilots are expected to feel the handling differences when performing the simulated flight with the airplanes having the servovalves setup and the on-off valves setup.

\section{CONCLUSION}

The main objective of the present work was to analyze the viability for the utilization of the SIVOR platform as a testbed for the evaluation of the F16 behavior changes when using on-off valves instead of the conventional servovalves. According to the presented results, it is possible to conclude that the SIVOR platform is expected to provide adequate motion perception for the pilots without reaching its workspace limits, therefore confirming that it has the potential for the proposed task. 


\section{ACKNOWLEDGEMENTS}

This study was financed in part by the Coordenação de Aperfeiçoamento de Pessoal de Nível Superior - Brasil (CAPES) - Finance Code 001 (Process 88881.122161/2016-01). The authors also acknowledge the financial support of CISB, FAPESP (Process 2012/51085-3) and CNPq (Process 303271/2017-5).
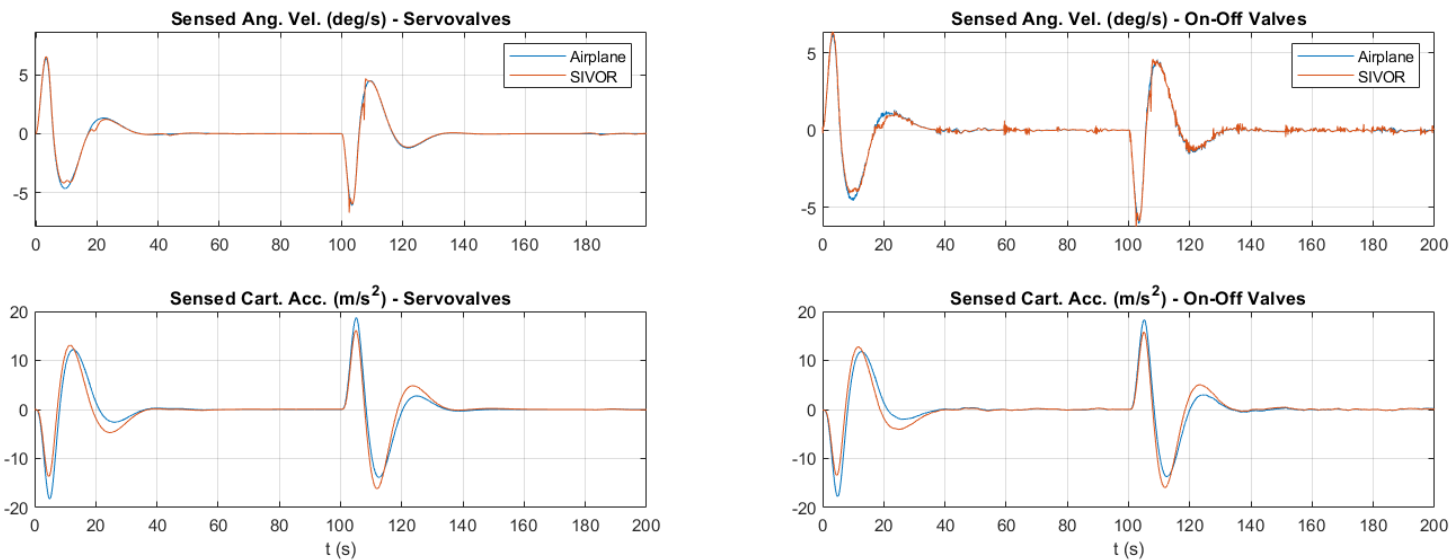

Figure 4 - Comparison between motion perceptions in the airplane and in the SIVOR platform
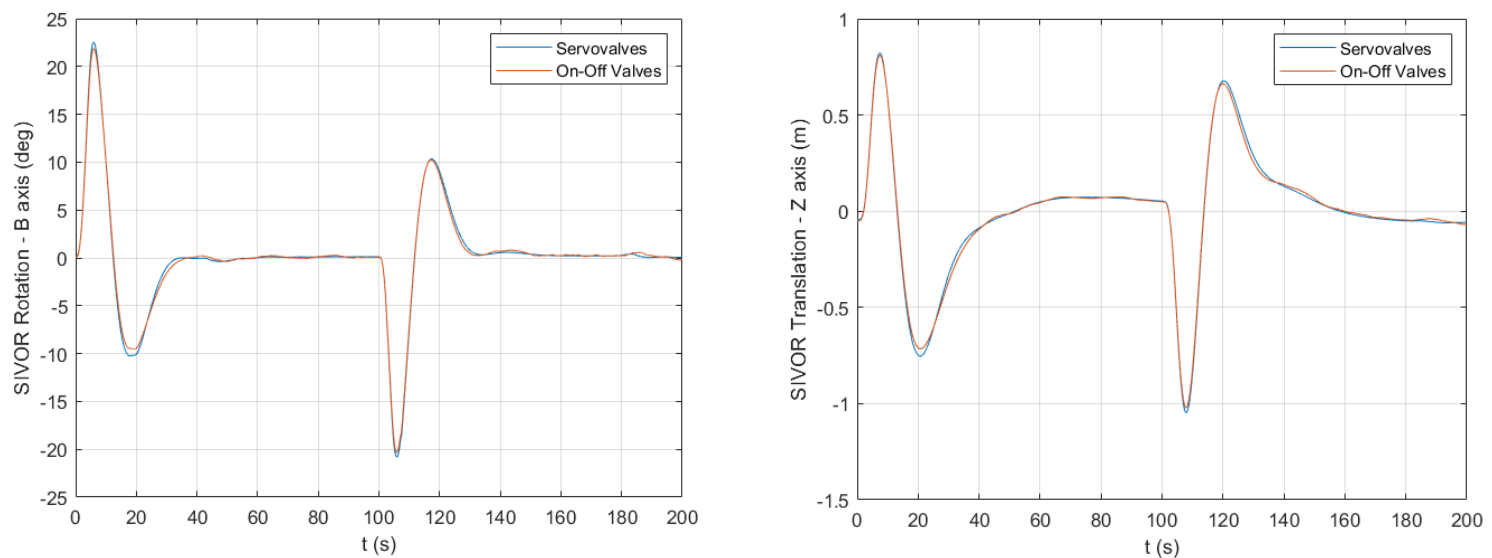

Figure 5 - Workspace usage of the SIVOR platform to simulate the proposed flight

\section{REFERENCES}

[1] Achten, P. "Convicted to innovation in fluid power", Proceedings of the Institute of Mechanical Engineers, Part I: Journal of Systems and Control Engineering. Volume 224, Number 6, pp 619-621, 2010.

[2] Locateli, C.C., Teixeira, P.L., De Pieri, E.R., Krus, P., De Negri, V.J. "Digital hydraulic system using pumps and on/off valves controlling the actuator", Proceedings of the 8th FPNI Ph.D Symposium on Fluid Power, Jun. 2014.

[3] Scheidl, R.; Linjama, M.; Schmidt, S. "Is the future of fluid power digital?", Proceedings of the Institution of Mechanical Engineers, Part I: Journal of Systems and Control Engineering, Volume 226, Number 6, pp. 721-723, 2012.

[4] Heitzig, S., Sgro, S., Theissen, H. "Energy efficiency of hydraulic systems with shared digital pumps", International Journal of Fluid Power, Volume 13, pp. 49-58, 2012.

[5] Linjama, M., Vihtanen, H.-P., Sipola, A., Vilenius, M. "Secondary controlled multi-chamber hydraulic cylinder", The 11th Scandinavian International Conference on Fluid Power. Linköping, Sweden, Jun. 2009.

[6] Huova, M.; Laamanen, A. "Control of three-chamber cylinder with digital valve system”, Proceedings of The Second Workshop on Digital Fluid Power, pp. 94-105, Nov. 2009. 
[7] Sartori-Natal, G., Hernandez-Arjoni, D., De Oliveira, W.R., Boulhosa-Rodamilans, G., Silveira, L., Da Silva, E.T., Villani, E., Gonzaga-Trabasso, L. "Implementation analysis of a washout filter on a robotic flight simulator - A case study”, Journal of Aerospace Technology and Management, Volume 11, 2019 (in press).

[8] Schmidt, S.F., Conrad, B. "Motion drive signals for piloted flight simulators”, NASA Report, CR-1601, 1970.

[9] Fernandez, C., Goldberg, P. "Physiology of peripheral neurons innervating semi-circular canals of the squirrel monkey II: Response to sinusoidal stimulation and dynamics of peripheral vestibular system”. Journal of Neurophysiology, Volume 34, Number 4, pp. 661-675, 1971.

[10] Telban, R., Cardullo, F. "Motion cueing algorithm development: Human-centered linear and nonlinear approaches”, NASA Langley Research Center. Technical Report CR-2005-213747, 2005. 